\title{
An adaptive threshold feedback compression scheme based on Channel Quality Indicator (CQI) in Long Term Evolution (LTE) system
}

\begin{abstract}
Channel quality indicator (CQI) feedback in long-term evolution (LTE) system is an essential technique in describing the instantaneous channel state information. The CQI calculations highly depend on the accuracy of the channel estimation process in order to assign appropriate modulation and coding scheme. However, one of the critical issues affecting the LTE system performance is obtaining the CQI for each transmission period which will inevitably cost many resources. Therefore, an appropriate method for reducing CQI feedback overhead along with accurate channel estimation technique is required to manage the allocated resources and obtains significant improvements in system performance. In this paper, an adaptive threshold feedback compression scheme based on CQI scheme is proposed to obtain better system performance in terms of system throughput and error rate in LTE system. This proposed adaptive scheme dynamically adapts its threshold level to the signal to noise ratio variations, thus increasing the throughput and reducing the CQI feedback overhead. Results show that the proposed CQI based adaptive threshold feedback compression scheme enhances the tradeoff between system throughput and block error rate.
\end{abstract}

Keyword: $\quad 3 G P P$ LTE; OFDM; MIMO; Channel quality indicator (CQI) feedback; Channel estimation 